\title{
Prostate cancer update from the American Society of Clinical Oncology (ASCO) annual meeting 2016
}

\author{
Anil Kapoor, MD, FRCSC \\ Professor of Surgery (Urology), McMaster University, Associate Editor (Oncology), Canadian Urological Association Journal (CUA), Hamilton, ON, Canada
}

Cite as: Can Urol Assoc J 2016;10(7-8-Supp|3):S137. hittp://dx.doi.org/10.5489/cuaj.4053

7 he American Society of Clinical Oncology (ASCO) annual meeting was held in Chicago, IL, from June 3-7, 2016. Over 30000 oncology professionals from around the world attended this meeting. This premier oncology event discussed state-of-the-art treatment modalities, new therapies, and controversies in oncology. In this report, we summarize the key prostate oncology research presented at the meeting.

For localized prostate cancer, abstracts on the role of magnetic resonance (MR) imaging for prostate cancer diagnosis are summarized, suggesting MR prostate imaging may prevent unnecessary prostate biopsies. In the radiation field, the PROFIT data was presented, suggesting shorter radiation fractionation is not inferior to conventional radiotherapy for intermediate-risk localized prostate cancer. The final analysis of GETUG 14 suggests the addition of short-term androgen depletion to high-dose radiotherapy improves clinical/ biochemical relapse-free survival in men with localized intermediate-risk prostate cancer. In the surgical arena, data is presented suggesting that statin use improves biochemical recurrent-free survival in men undergoing radical prostatectomy, as well as abstracts suggesting radical prostatectomy is associated with better long-term survival, but higher early morbidity compared to external beam radiation therapy (EBRT) in the treatment of Stage II prostate cancer,

For metastatic prostate cancer, abstracts are summarized on early treatment with docetaxel in metastatic hormonesensitive prostate cancer ( $\mathrm{mHSPC}$ ) patients and quality of life endpoints offset by long-term gains in overall survival (OS), as well as data on intermittent docetaxel being noninferior to continuous docetaxel in men with metastatic castrate-resistant prostate cancer (mCRPC) in a small sample size study. The FIRSTANA study is summarized, comparing cabazitaxel/prednisone with docetaxel/prednisone in chemotherapy-naive mCRPC and demonstrating similar OS.

Abstracts on radionuclides in prostate cancer treatment are summarized, including data on re-treatment with radium-223 after initial six cycles, hypothesis-generating there may be some additional benefit in patients who have progressed on the initial six cycles of radium-223. Combination of radium-223 with docetaxel appears to be well-tolerated and improves time to prostate-specific antigen (PSA) progression and progression-free survival in men with mCRPC. Dr. Fred Saad presented data from the radium-223 EAP, showing those patients who receive radium-223 earlier in the course of prostate cancer treatment may increase the likelihood of receiving a full course of treatment.

Finally, we present new data on androgen receptor (AR) axis-targeted agents, specifically with abiraterone and enzalutamide. PSA declines with enzalutamide are associated with clinical benefit and improved OS in mCRPC patients, and lack of PSA declines may be used to identify men with early resistance. We also summarize abstracts examining new data on the cognitive impairment and central nervous system toxicities with AR-targeted therapies and the need for further research in this domain.

Competing interests: None of the authors have received any honoraria for preparation of this ASCO 2016 report.

Acknowledgememts: The authors would like to thank Science \& Medicine Canada Inc. for editorial assistance with this report.

Correspondence: Dr. Anil Kapoor, McMaster University, Hamilton, ON, Canada; akapoor@mcmaster.ca 